\title{
miR-202-5p inhibits the migration and invasion of osteosarcoma cells by targeting ROCK1
}

\author{
CONGDA LI, DEYING MA, JINHU YANG, XIANGBO LIN and BO CHEN \\ Department of Orthopedic, People's Hospital of Rizhao, Rizhao, Shandong 276800, P.R. China
}

Received March 2, 2018; Accepted May 2, 2018

DOI: $10.3892 / 01.2018 .8694$

\begin{abstract}
Many studies have shown that microRNA regulates the development and treatment of osteosarcoma (OS). In many human cancer studies, the expression of microRNA-202 has been shown to be abnormal. The aim of the study was to examine the role of miR-202-5p in the occurrence and formation of OS. miR-202-5p and Rho-associated coiled-coil containing protein kinase 1 (ROCK1) levels were assessed using RT-qPCR in OS tissues and cell lines. The cell migrating and invasive abilities were detected by the Transwell assay in OS. Moreover, the relationship between miR-202-5p and ROCK1 was verified via luciferase reporter assay. The protein level of ROCK1 was identified by western blot analysis. Downregulation of miR-202-5p was identified in OS tissues and cell lines. In addition, the miR-202-5p overexpression had inhibitory action for cell migration and invasion in OS. Moreover, miR-202-5p directly targeted ROCK1 and negatively regulated its expression. Upregulation of ROCK1 had a carcinogenic effect in OS. Furthermore, the upregulation of ROCK1 restored the suppressive effect of miR-202-5p. miR-202-5p, in turn, weakened the abilities of cell migration and invasion in OS by inhibiting ROCK1 expression. As a result, miR-202-5p may be developed as a potential pathway in the reatment of OS.
\end{abstract}

\section{Introduction}

Osteosarcoma (OS) is a common malignant bone tumor occurring in children or teenagers younger than 20 years (1). More specifically, OS usually occurs in children with bone malignancy, accounting for almost $5 \%$ of all pediatric tumors (2). Moreover, incidence of OS in primary malignant tumor ranks first, showing a very high tumor malignant degree and poor prognosis of OS patients (3). In addition, OS can spread to the lungs within a few months, resulting

Correspondence to: Dr Jinhu Yang, Department of Orthopedic, People's Hospital of Rizhao, 126 Tai'an Road, Donggang, Rizhao, Shandong 276800, P.R. China

E-mail: yh94xw@163.com

Key words: miR-202-5p, invasion, migration, ROCK1, osteosarcoma in a 3 - to 5-year survival rate of only 5-20\% after amputation (4). Thus, finding effective biomarkers for the diagnosis and therapy of OS is required.

MicroRNAs (miRNAs) have been reported to be unusually upregulated or downregulated in various human cancers. For instance, miR-21 was upregulated in colorectal cancer (5), while miR-126 was downregulated in lung cancer (6). Moreover, the dysregulated expression of miRNAs was also closely-associated with the pathogenesis and development of cancers (7). In OS, downregulation of miR-375, miR-302b and miR-216a was detected in previous studies (8-10). Furthermore, the upregulation of miR-19, miR-92b and miR-146a was found in OS (11-13). Therefore, miRNAs participated in the tumorigenesis of OS, as indicated in previous studies. Specifically, the decrease of miR-202 expression was identified in gastric cancer (14), colorectal carcinoma (15) and endometrial adenocarcinoma (16). Those studies indicated that miR-202 also affects the progression of OS. However, the specific function of miR-202 in OS has yet to be analysed.

Rho-associated coiled-coil containing protein kinase 1 (ROCK1) is considered a direct target gene of many miRNAs in various kinds of human cancers, such as miR-124 (17), miR-144 (18) and miR-148a (19). Moreover, the carcinogenesis of ROCK1 was identified in glioma (20), breast cancer (21) and non-small cell lung cancer (22). In OS, ROCK1 was employed as a potential therapeutic target (23). However, the relationship between ROCK1 and miR-202 in OS still remains unclear.

In the present study, we aimed to investigate the specific effect of miR-202-5p in OS as well as the interaction between miR-202-5p and ROCK1. Finally, we examined whether miR-202-5p weakened the abilities of cell migration and invasion in OS by inhibiting ROCK1 expression.

\section{Materials and methods}

Clinical tissues. Thirty-six surgical tumor specimens and adjacent tissue samples were obtained from the People's Hospital of Rizhao (Rizhao, China) after receiving written informed consent. The patients received no treatment prior to surgery. Human tissue was frozen in liquid nitrogen and then stored in a refrigerator at $-80^{\circ} \mathrm{C}$ for further experiments. This experiment was approved by the Institutional Ethics Committee of People's Hospital of Rizhao. 
Cell cultures and cell transfection. The human OC cell lines U2OS, MG-63, HOS and human normal osteoblast cell line hFOB1.19 were used for this experiment. All the cell lines came from the American Type Culture Collection (ATCC, Manassas, VA, USA). These cells were seeded in DMEM or RPMI-1640 medium containing 10\% fetal bovine serum (FBS) and cultured at $37^{\circ} \mathrm{C}$ with $5 \% \mathrm{CO}_{2}$.

The miR-202-5p mimic and inhibitor, ROCK1 siRNA (si-ROCK1) were obtained from GenePharma Co., Ltd. (Shanghai, China). Then they were transferred into HOS cells using Lipofectamine 2000 (Invitrogen; Thermo Fisher Scientific, Inc., Waltham, MA, USA) based on the manufacturer's protocols.

$R T$-qPCR. TRIzol reagent (Invitrogen; Thermo Fisher Scientific, Inc.) was applied to extract total RNA containing miRNA to quantify miR-202-5p expression in OS tissues and cell lines. RT-qPCR was carried out using the SYBR-Green Master Mix (Toyobo Co., Ltd., Osaka, Japan) on Applied Biosystems 7500 Sequence detection system (Applied Biosystems; Thermo Fisher Scientific, Inc.). U6 and GAPDH were used as the control for miR-202-5p and ROCK1. Expression was calculated using the $2^{-\Delta \Delta \mathrm{Cq}}$ method.

Dual luciferase reporter assay. 293T cells were incubated in 24-well plates for the Dual-Luciferase Reporter Assay System (Promega Corporation, Madison, WI, USA). The wild or mutant type of 3'-untranslated region (3'-UTR) of ROCK1 was inserted into the pGL3 promoter vector (Invitrogen; Thermo Fisher Scientific,Inc.) for luciferase reporter experiments. Then, wild or mutant type of 3'-UTR of ROCK1 and miR-202-5p mimic was transfected into 293T cells. Subsequently, the Dual Luciferase Reporter Assay System (Promega Corporation) was applied to measure luciferase activities.

Transwell assay. The Transwell chamber (24-well) was employed to perform cell migration and invasion assays. HOS cells $\left(5 \times 10^{4}\right)$ without serum were placed in the upper chamber on the non-coated membrane, and the lower chamber was filled with $10 \%$ FBS to induce HOS cells to migrate or invade through the membrane. In addition, the cells were placed in the upper chamber with the matrigel (BD Biosciences, Franklin Lakes, NJ, USA) for the invasion assay. Then, the cells were incubated for the migration and invasion assay. Finally, the cells were stained with crystal violet. The number of cells was counted using an inverted light microscope (Zeiss, Oberkochen, Germany).

Western blot analysis. The protein samples were obtained using RIPA lysis buffer. Proteins were separated through a $10 \%$ SDS-PAGE and incubated with 5\% non-fat milk in PVDF membranes at room temperature. Next, we incubated the membranes overnight at $4^{\circ} \mathrm{C}$ with mouse anti-ROCK $1(1: 1,000$, rabbit polyclonal antibody, ab97592), anti-GAPDH (1:1,000, rabbit polyclonal antibody, ab9485), which were subsequently incubated with goat anti-mouse secondary antibodies. Then, goat anti-rabbit IgG H\&L secondary antibodies $(1: 1,000$, goat polyclonal second antibody, ab150077) protein levels were measured using enhanced chemiluminescence (ECL; Pierce; Thermo Fisher Scientific, Inc.).
Statistical analysis. The obtained data were shown as the mean \pm SD. The data were analyzed with GraphPad Prism 6.0. (GraphPad Software Inc., La Jolla, CA, USA). The difference was calculated according to the Chi-square or ANOVA followed by a Tukey's test. $\mathrm{P}<0.05$ was considered statistically significant.

\section{Results}

Low expression of miR-202-5p is identified in OS tissues and cell lines. The miR-202-5p level was detected in OS tissues via RT-qPCR (Fig. 1A). The lower expression of miR-202-5p was identified in OS tissues in comparison with the normal tissues. Moreover, downregulation of miR-202-5p was found in OS that had lymph-node metastasis (Fig. 1B). It indicated that the aberrant expression was related to lymph-node metastasis. Similarly, miR-202-5p downregulation was also assessed in U2OS, MG-63 and HOS cell lines, except for the human osteoblast cell line hFOB1.19 (Fig. 1C). In brief, downregulation of miR-202-5p may play a vital role in the metastasis of OS.

Overexpression of miR-202-5p exerts an inhibitory effect on cell migration and invasion in OS. miR-202-5p mimic or inhibitor was transfected into HOS cells to investigate its function in OS. The miR-202-5p levels were measured in these transfected cells using RT-qPCR (Fig. 2A). Then the migrating and invasive abilities in these transfected cells were detected by the Transwell assay. As expected, the migrating and invasive abilities were reduced by miR-202-5p mimics but enhanced by miR-202-5p inhibitor in OS cells (Fig. 2B and C). These findings showed that miR-202-5p as a suppressive miRNA exerts an inhibitory effect on cell migration and invasion in OS.

miR-202-5p directly targets ROCK1 and negatively regulates its expression. Then we performed luciferase reporter assays to confirm the prediction that miR-202-5p binds to the 3'-UTR of ROCK1 (Fig. 3A). As predicted, co-transfection of wild-type of ROCK1 and miR-202-5p mimic in 293T cells reduced the luciferase activity and no change was found in cells containing the mutant type of ROCK1 and miR-202-5p mimic compared to the negative control (Fig. 3B). In addition, the ROCK1 levels in U2OS, MG-63, HOS and hFOB1.19 cell lines were identified via RT-qPCR. Upregulation of ROCK1 was found in U2OS, MG-63, and HOS cells apart from hFOB1.19 (Fig. 3C). Furthermore, we observed the ROCK1 level in cells with miR-202-5p mimic or inhibitor to further explore their relationship. We found that ROCK1 level was declined by the miR-202-5p mimic and enhanced by the miR-202-5p inhibitor by RT-qPCR and western blot analysis (Fig. 3D). Collectively, miR-202-5p directly targeted ROCK1 and negatively regulated its expression.

ROCK1 has a carcinogenic effect on OS cells. Next, ROCK1 siRNA was transfected into HOS cells to block its expression. Moreover, the mRNA and protein level of ROCK1 were declined by ROCK1 siRNA in HOS cells (Fig. 4A and B). Similarly, the ROCK1 siRNA also impaired the migrating and invasive abilities in HOS cells, which was the same as the inhibitory action of miR-202-5p overexpression (Fig. 4C and D). In brief, ROCK1 had a carcinogenic effect on OS. 
A ลำ

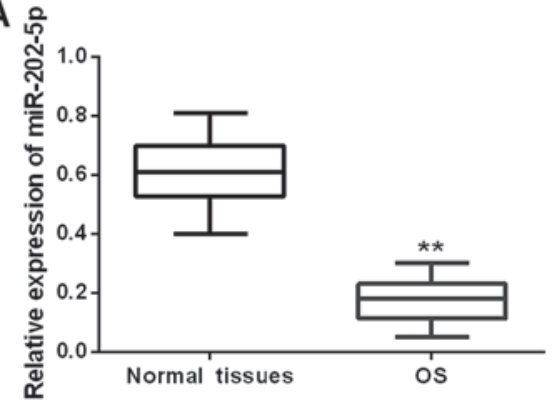

B 。

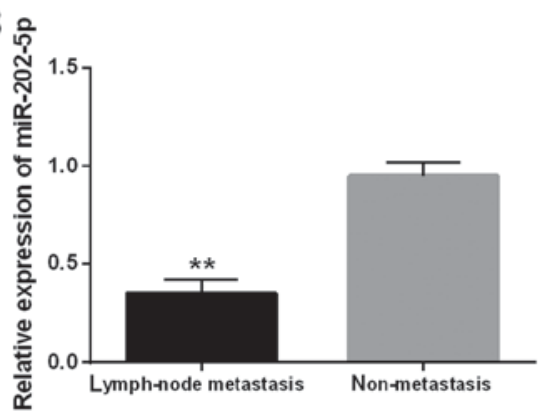

$C$ 。

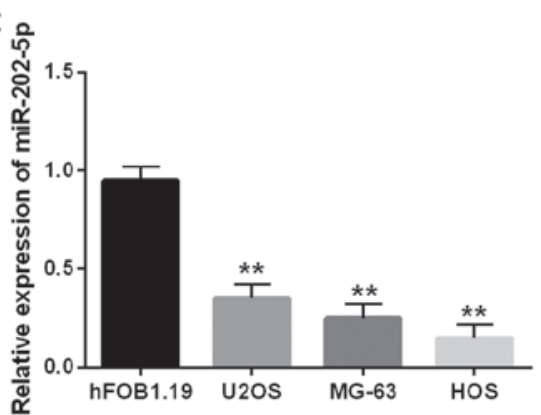

Figure 1. Downregulation of miR-202-5p was identified in OS tissues and cell lines. (A) The miR-202-5p level in OS tissues and normal tissues was detected via RT-qPCR. (B) miR-202-5p level in OS cells with lymph-node metastasis and non-metastasis. (C) miR-202-5p expression in U2OS, MG-63, HOS and human normal osteoblast cell line hFOB1.19. ${ }^{* *} \mathrm{P}<0.01$. OS, osteosarcoma.

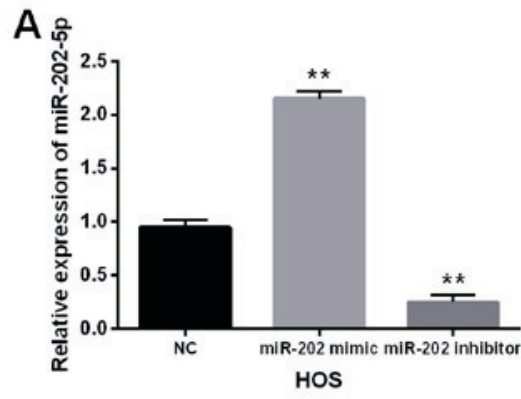

B
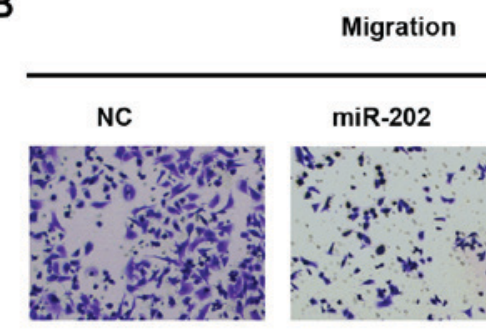

miR-202
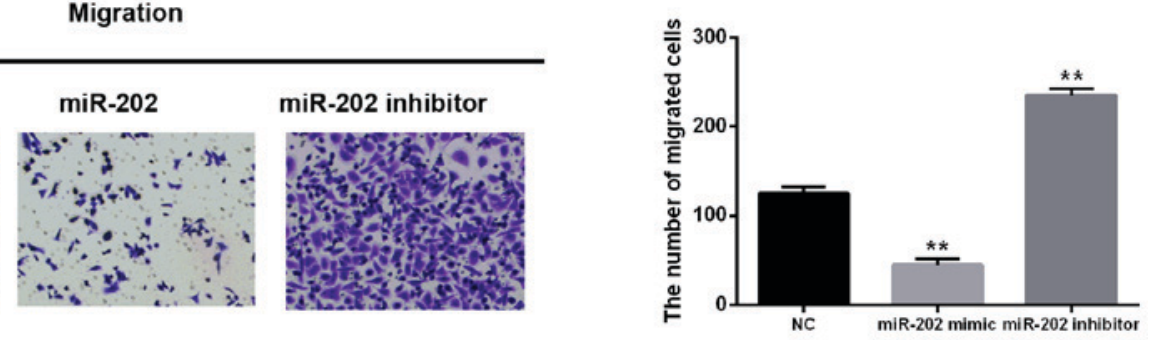

C

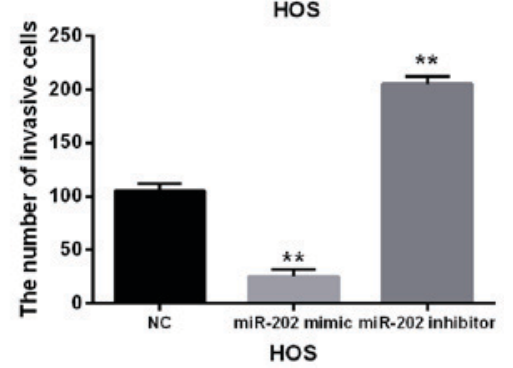

Figure 2. Overexpression of miR-202-5p had an inhibitory effect on cell migration and invasion in OS. (A) miR-202-5p expression was examined in HOS cells containing miR-202-5p mimic or inhibitor via RT-qPCR. (B and C) The cell migration and invasion were measured in cells containing miR-202-5p mimic or inhibitor via the Transwell analysis. ${ }^{* *} \mathrm{P}<0.01$. OS, osteosarcoma.

Upregulation of ROCK1 restores the suppressive effect of miR-202-5p in OS. miR-202-5p mimic and ROCK1 vector were transfected into HOS cells to further investigate their suppressive function. Overexpression of ROCK1 recovered the decrease of ROCK1 mRNA and protein levels induced by miR-202-5p mimic (Fig. 5A and B). Functionally, the abilities of migration and invasion in transfected HOS cells containing miR-202-5p mimic and ROCK1 vector were regained in comparison with the cells only miR-202-5p mimic (Fig. 5C and D). Generally speaking, upregulation of
ROCK1 restored the suppressive effect of miR-202-5p in OS cells, which further indicated that miR-202-5p repressed cell migration and invasion in OS by regulating ROCK1.

\section{Discussion}

Previous studies have demonstrated that identification of miRNAs can be used as a biomarker for the diagnosis and prognosis of OS (24). In our study, downregulation of miR-202-5p and the upregulation of ROCK1 were identified 


\section{A}

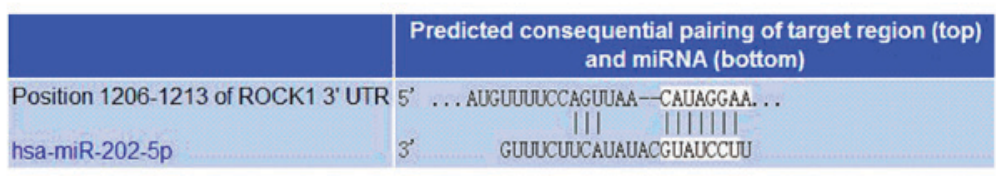

B

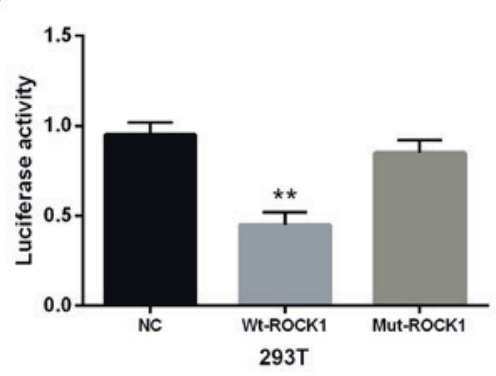

D

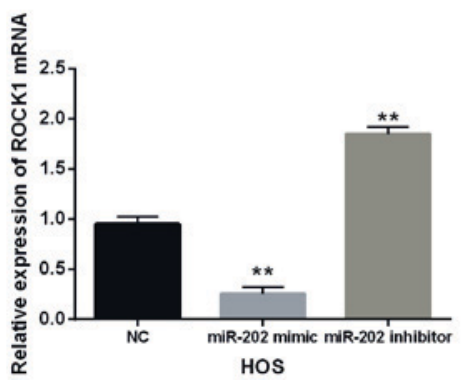

C

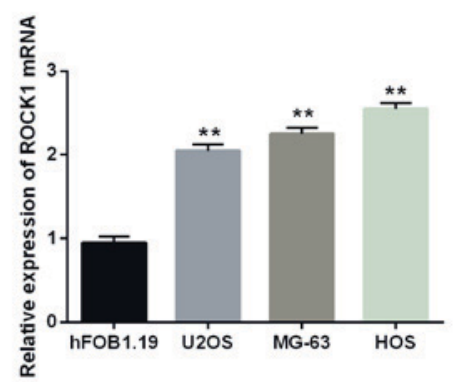

Figure 3. miR-202-5p directly targeted ROCK1 and negatively regulated its expression. (A) The binding sites of miR-202-5p on the 3'-UTR of ROCK1. (B) Luciferase reporter assay. (C) ROCK1 expression in U2OS, MG-63, HOS and hFOB1.19 cells (control). (D) mRNA and protein expressions of ROCK1 were assessed in cells containing miR-202-5p mimic or inhibitor. ${ }^{* *} \mathrm{P}<0.01$. ROCK1, Rho-associated coiled-coil containing protein kinase 1; 3'-UTR, 3'-untranslated region.

A

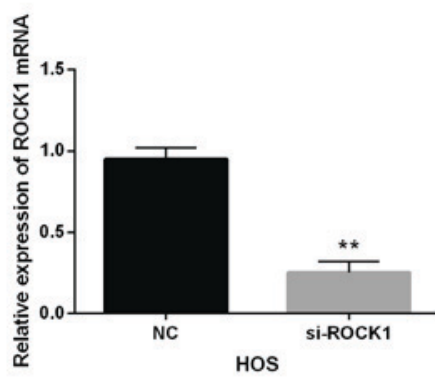

C

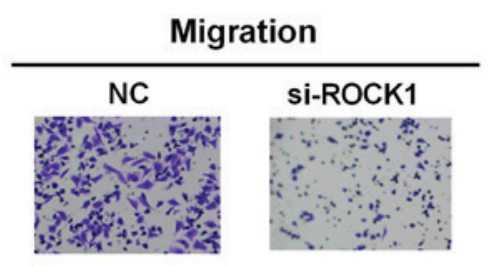

D

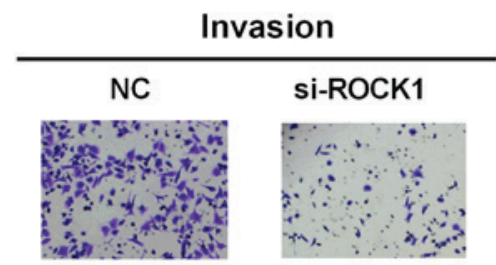

B

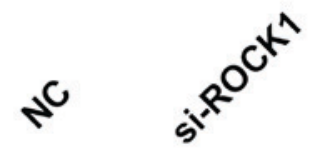

ROCK1

GAPDH
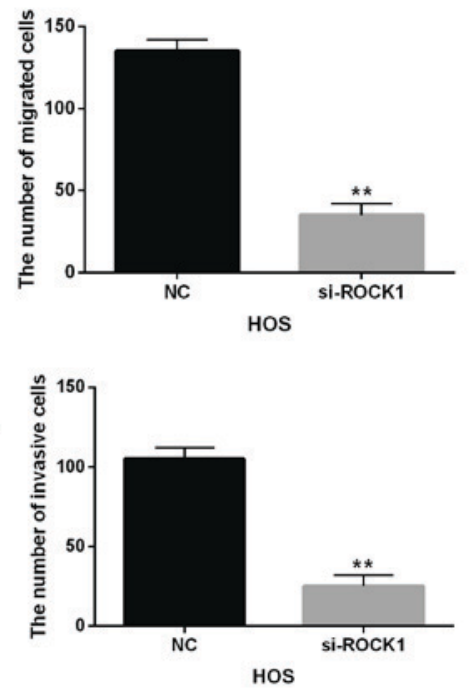

Figure 4. ROCK1 had a carcinogenic effect in OS cells. (A and B) The mRNA and protein expressions of ROCK1 were measured in cells containing ROCK1 siRNA. (C and D) Cell migration and invasion analysis of HOS cells with si-ROCK1 was detected by the Transwell assay. ${ }^{* *} \mathrm{P}<0.01$. OS, osteosarcoma; ROCK1, Rho-associated coiled-coil containing protein kinase 1; si-ROCK1, ROCK1 siRNA. 
A

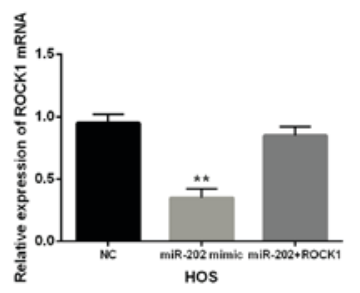

B

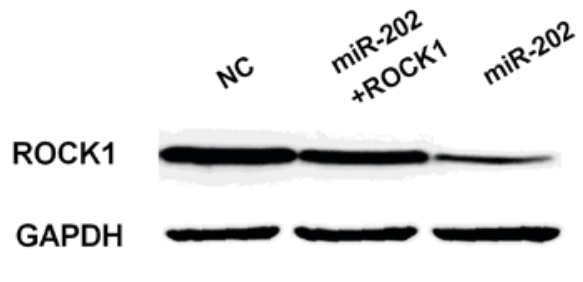

C
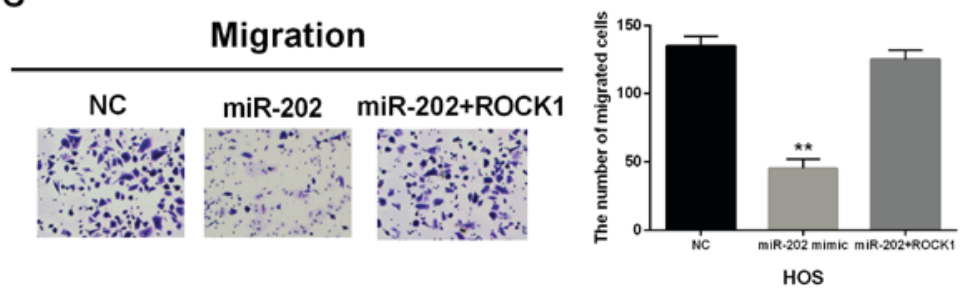

D

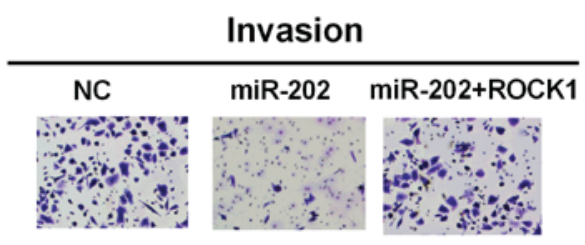

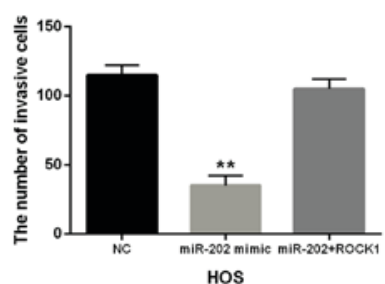

Figure 5. Upregulation of ROCK1 restored the inhibitory effect of miR-202-5p in OS. (A and B) The mRNA and protein expressions of ROCK1 were measured in cells containing miR-202-5p and ROCK1 vector. (C and D) The cell migration and invasion in cells containing ROCK1 vector and miR-202-5p were detected by the Transwell assay. ${ }^{* *} \mathrm{P}<0.01$. OS, osteosarcoma; ROCK1, Rho-associated coiled-coil containing protein kinase 1.

in OS. Overexpression of miR-202-5p impaired the migrating and invasive abilities in OS, which was similar to the knockout of ROCK1. Furthermore, the upregulation of ROCK1 restored the inhibitory effect of miR-202-5p in OS.

Many studies have shown that miR-202 was usually downregulated and participated in the formation of many human cancers. For instance, the miR-202 level was reduced and suppressed tumor progression in esophageal squamous cell carcinoma (25). In hepatocellular carcinoma, the miR-202 overexpression suppressed cell proliferation (26). Additionally, miR-202 was found to function as a suppressive miRNA in non-small cell lung cancer (27). The miR-202 downregulation was frequently identified in human cancers which was in agreement with our results in OS. More importantly, miR-202 was reported to be significantly declined. Moreover, it repressed cell growth and promoted cell apoptosis in OS (28). This study also confirmed our findings of miR-202 in OS. However, to the best of our knowledge, there was no study about the role of miR-202 for migration and invasion in OS cells. In addition, we demonstrated that the miR-202-5p overexpression had an inhibitory effect on cell migration and invasion in OS. Therefore, miR-202 may be used as an indicator for the diagnosis and prediction of OS, which was helpful in the treatment of OS patients.

To further explore the function of miR-202, ROCK1 was verified as a direct target of miR-202 in OS. To date, ROCK1 as a direct target gene has been reported to bind to miR-300 (29), miR-340 (30), miR-584 (31) and miR-1280 (32). However, the relationship between miR-202 and ROCK1 has not been analysed thus far. In the present study, upregulation and carcinogenic effects of ROCK1 were observed in OS. The same findings of ROCK1 were also reported in OS induced by miR-145 (33), miR-198 (34) and miR-335 (35). Findings of those studies were in agreement with our results. In addition, miR-202-5p was negatively associated with ROCK1 expression. Upregulation of ROCK1 restored the suppressive effect of miR-202-5p in OS. Collectively, miR-202-5p impaired the migrating and invasive abilities in OS partly by inhibiting ROCK1. Therefore, understanding the role of miR-202 is significant for the treatment of OS.

In conclusion, downregulation of miR-202-5p and upregulation of ROCK1 were found in OS. miR-202-5p was verified to directly target ROCK1. More importantly, miR-202-5p was identified to inhibit the migrating and invasive abilities in OS cells by inhibiting ROCK1.

\section{Acknowledgements}

Not applicable.

\section{Funding}

No funding was received.

\section{Availability of data and materials}

The datasets used and/or analyzed during the present study are available from the corresponding author on reasonable request. 


\section{Authors' contributions}

CL as the first author contributed significantly in the analysis of and wrote the manuscript. DM as the second author performed the data analyses and wrote the manuscript. XL as the fourth author helped perform the analysis with constructive discussions. BC as the fifth author sorted out experimental data. JY as the corresponding author contributed to the conception of the study. All authors read and approved the final manuscript.

\section{Ethics approval and consent to participate}

The study was approved by the Ethics Committee of the People's Hospital of Rizhao (Rizhao, China). Signed written informed consents were obtained from the patients and/or guardians.

\section{Consent for publication}

Not applicable.

\section{Competing interests}

The authors declare that they have no competing interests.

\section{References}

1. Mirabello L, Troisi RJ and Savage SA: Osteosarcoma incidence and survival rates from 1973 to 2004: Data from the Surveillance, Epidemiology, and End Results Program. Cancer 115: 1531-1543, 2009.

2. Allison DC, Carney SC, Ahlmann ER, Hendifar A, Chawla S, Fedenko A, Angeles C and Menendez LR: A meta-analysis of osteosarcoma outcomes in the modern medical era. Sarcoma 2012: 704872, 2012.

3. Ottaviani G and Jaffe N: The epidemiology of osteosarcoma. Cancer Treat Res 152: 3-13, 2009.

4. Lee JA, Kim DH, Lim JS, Park KD, Song WS, Lee SY and Jeon DG: The survival of osteosarcoma patients 10 years old or younger is not worse than the survival of older patients: A retrospective analysis. Cancer Res Treat 39: 160-164, 2007.

5. Asangani IA, Rasheed SA, Nikolova DA, Leupold JH, Colburn NH, Post S and Allgayer H: MicroRNA-21 (miR-21) post-transcriptionally downregulates tumor suppressor Pdcd4 and stimulates invasion, intravasation and metastasis in colorectal cancer. Oncogene 27: 2128-2136, 2008.

6. Liu B, Peng XC, Zheng XL, Wang J and Qin YW: MiR-126 restoration down-regulate VEGF and inhibit the growth of lung cancer cell lines in vitro and in vivo. Lung Cancer 66: 169-175, 2009.

7. Iorio MV, Visone R, Di Leva G, Donati V, Petrocca F, Casalini P, Taccioli C, Volinia S, Liu CG, Alder H, et al: MicroRNA signatures in human ovarian cancer. Cancer Res 67: 8699-8707, 2007

8. Liu W, Zhao X, Zhang YJ, Fang GW and Xue Y: MicroRNA-375 as a potential serum biomarker for the diagnosis, prognosis, and chemosensitivity prediction of osteosarcoma. J Int Med Res 46: 975-983, 2018

9. Xie Y, Sun W, Deng Z, Zhu X, Hu C and Cai L: miR-302b suppresses osteosarcoma cell migration and invasion by targeting Runx2. Sci Rep 7: 13388, 2017.

10. Ji Q, Xu X, Li L, Goodman SB, Bi W, Xu M, Xu Y, Fan Z, Maloney WJ, Ye Q, et al: miR-216a inhibits osteosarcoma cell proliferation, invasion and metastasis by targeting CDK14. Cell Death Dis 8: e3103, 2017.

11. Sun Z, Liu Q, Hong H, Zhang $\mathrm{H}$ and Zhang T: miR-19 promotes osteosarcoma progression by targeting SOCS6. Biochem Biophys Res Commun 495: 1363-1369, 2018.

12. Wu Q,Zhou W, Feng Q, Liu X, Xiong Y and Li H: MicroRNA-92b promotes cell proliferation and invasion in osteosarcoma by directly targeting Dickkopf-related protein 3. Exp Ther Med 15: 173-181, 2018.

13. Zhou C, Jiang CQ,Zong Z, Lin JC and Lao LF: miR-146a promotes growth of osteosarcoma cells by targeting ZNRF3/GSK-3 $\beta$ / $\beta$-catenin signaling pathway. Oncotarget 8: 74276-74286, 2017.
14. Zhao Y, Li C, Wang M, Su L, Qu Y, Li J, Yu B, Yan M, Yu Y, Liu B, et al: Decrease of miR-202-3p expression, a novel tumor suppressor, in gastric cancer. PLoS One 8: e69756, 2013.

15. Wang Q, Huang Z, Guo W, Ni S, Xiao X, Wang L, Huang D, Tan C, Xu Q, Zha R, et al: microRNA-202-3p inhibits cell proliferation by targeting ADP-ribosylation factor-like $5 \mathrm{~A}$ in human colorectal carcinoma. Clin Cancer Res 20: 1146-1157, 2014.

16. Deng X, Hou C, Liang Z, Wang H, Zhu L and Xu H: miR-202 suppresses cell proliferation by targeting FOXR2 in endometrial adenocarcinoma. Dis Markers 2017: 2827435, 2017.

17. Xu X, Li S, Lin Y, Chen H, Hu Z, Mao Y, Xu X, Wu J, Zhu Y, Zheng $X$, et al: MicroRNA-124-3p inhibits cell migration and invasion in bladder cancer cells by targeting ROCK1. J Transl Med 11: 276, 2013.

18. Cai SD, Chen JS, Xi ZW, Zhang LJ, Niu ML and Gao ZY: MicroRNA 144 inhibits migration and proliferation in rectal cancer by downregulating ROCK 1. Mol Med Rep 12: 7396-7402, 2015.

19. Zheng B, Liang L, Wang C, Huang S, Cao X, Zha R, Liu L, Jia D, Tian Q, Wu J, et al: MicroRNA-148a suppresses tumor cell invasion and metastasis by downregulating ROCK1 in gastric cancer. Clin Cancer Res 17: 7574-7583, 2011.

20. An L, Liu Y, Wu A and Guan Y: microRNA-124 inhibits migration and invasion by down-regulating ROCK1 in glioma. PLoS One 8: e69478, 2013.

21. Li J, Song Y, Wang Y, Luo J and Yu W: MicroRNA-148a suppresses epithelial-to-mesenchymal transition by targeting ROCK1 in non-small cell lung cancer cells. Mol Cell Biochem 380: 277-282, 2013.

22. Zheng M, Sun X, Li Y and Zuo W: MicroRNA-145 inhibits growth and migration of breast cancer cells through targeting oncoprotein ROCK1. Tumour Biol 37: 8189-8196, 2016.

23. Liu X, Choy E, Hornicek FJ, Yang S, Yang C, Harmon D, Mankin H and Duan Z: ROCK1 as a potential therapeutic target in osteosarcoma. J Orthop Res 29: 1259-1266, 2011.

24. Cong C, Wang W, Tian J, Gao T, Zheng W and Zhou C: Identification of serum miR-124 as a biomarker for diagnosis and prognosis in osteosarcoma. Cancer Biomark 21: 449-454, 2018.

25. Meng X, Chen X, Lu P, Ma W, Yue D, Song L and Fan Q: MicroRNA-202 inhibits tumor progression by targeting LAMA1 in esophageal squamous cell carcinoma. Biochem Biophys Res Commun 473: 821-827, 2016.

26. Zhang Y, Zheng D, Xiong Y, Xue C, Chen G, Yan B and Ye Q: miR-202 suppresses cell proliferation in human hepatocellular carcinoma by downregulating LRP6 post-transcriptionally. FEBS Lett 588: 1913-1920, 2014

27. Zhao Z, Lv B, Zhang L, Zhao N and Lv Y: miR-202 functions as a tumor suppressor in non-small cell lung cancer by targeting STAT3. Mol Med Rep 16: 2281-2289, 2017.

28. Sun Z, Zhang T, Hong H, Liu Q and Zhang H: miR-202 suppresses proliferation and induces apoptosis of osteosarcoma cells by downregulating Gli2. Mol Cell Biochem 397: 277-283, 2014.

29. Zhou F, Li Y, Hao Z, Liu X, Chen L, Cao Y, Liang Z, Yuan F, Liu J, Wang J, et al: MicroRNA-300 inhibited glioblastoma progression through ROCK1. Oncotarget 7: 36529-36538, 2016.

30. Maskey N, Li D, Xu H, Song H, Wu C, Hua K, Song J and Fang L: MicroRNA-340 inhibits invasion and metastasis by downregulating ROCK1 in breast cancer cells. Oncol Lett 14: 2261-2267, 2017.

31. Xue H, Guo X, Han X, Yan S, Zhang J, Xu S, Li T, Guo X, Zhang P, Gao X, et al: MicroRNA-584-3p, a novel tumor suppressor and prognostic marker, reduces the migration and invasion of human glioma cells by targeting hypoxia-induced ROCK1. Oncotarget 7: 4785-4805, 2016.

32. Majid S, Dar AA, Saini S, Shahryari V, Arora S, Zaman MS, Chang I, Yamamura S, Chiyomaru T, Fukuhara S, et al: MicroRNA-1280 inhibits invasion and metastasis by targeting ROCK1 in bladder cancer. PLoS One 7: e46743, 2012.

33. Lei P, Xie J, Wang L, Yang X, Dai Z and Hu Y: microRNA-145 inhibits osteosarcoma cell proliferation and invasion by targeting ROCK1. Mol Med Rep 10: 155-160, 2014.

34. Zhang S, Zhao Y and Wang L: MicroRNA-198 inhibited tumorous behaviors of human osteosarcoma through directly targeting ROCK1. Biochem Biophys Res Commun 472: 557-565, 2016.

35. Wang Y, Wang N, Zeng X, Sun J, Wang G, Xu H and Zhao W: MicroRNA-335 and its target Rock1 synergistically influence tumor progression and prognosis in osteosarcoma. Oncol Lett 13: 3057-3065, 2017.

This work is licensed under a Creative Commons Attribution-NonCommercial-NoDerivatives 4.0 International (CC BY-NC-ND 4.0) License. 